\title{
Expert Opinions on the Current Therapeutic Management of Inflammatory Bowel Disease during the COVID-19 Pandemic: Japan IBD COVID-19 Taskforce, Intractable Diseases, the Health and Labor Sciences Research
}

\author{
Hiroshi Nakase ${ }^{a}$ Takayuki Matsumoto $^{b}$ Minoru Matsuurac Hideki lijima ${ }^{d}$ \\ Katsuyoshi Matsuoka ${ }^{\text {e Naoki Ohmiyaf }}{ }^{f}$ Shunji Ishiharag Fumihito Hirai ${ }^{\text {h }}$ \\ Kouhei Wagatsuma ${ }^{a}$ Yoshihiro Yokoyama ${ }^{a}$ Tadakazu Hisamatsu $^{c}$ \\ aDepartment of Gastroenterology and Hepatology, Sapporo Medical University School of Medicine, Sapporo, Japan; \\ ${ }^{b}$ Division of Gastroenterology, Department of Medicine, Iwate Medical University, Morioka, Japan; 'Department \\ of Gastroenterology and Hepatology, Kyorin University School of Medicine, Tokyo, Japan; dDepartment of \\ Gastroenterology and Hepatology, Osaka University Graduate School of Medicine, Osaka, Japan; 'Department \\ of Gastroenterology and Hepatology, Toho University Sakura Medical Center, Chiba, Japan; ' Department of \\ Gastroenterology, Fujita Health University School of Medicine, Aichi, Japan; ${ }^{9}$ Department of Gastroenterology,

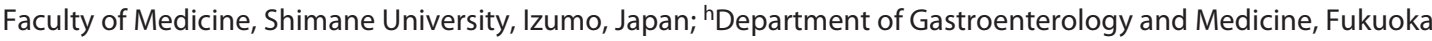 \\ University Faculty of Medicine, Fukuoka, Japan
}

\section{Keywords}

Coronavirus infectious disease 2019 . Severe acute respiratory syndrome coronavirus 2 - Inflammatory bowel disease $\cdot$ Steroid $\cdot$ Immunomodulators $\cdot$ Biologics

\begin{abstract}
Background: The COVID-19 pandemic, caused by severe acute respiratory syndrome coronavirus 2 (SARS-CoV-2), has emerged as a dramatic challenge for all healthcare systems worldwide. This outbreak immediately affected gastroenterologists as well as global physicians worldwide because COVID-19 can be associated with not only triggering respiratory inflammation but also gastrointestinal (Gl) inflammation based on the mechanism by which SARS-CoV-2 enters cells via its receptor the angiotensin-converting enzyme 2, which is expressed on Gl cells. However, the comorbidity
\end{abstract}

spectrum of digestive system in patients with COVID-19 remains unknown. Because the inflammatory bowel disease (IBD) management involves treating uncontrolled inflammation with immune-based therapies, physicians, and patients have great concern about whether IBD patients are more susceptible to SARS-CoV-2 infection and have worsened disease courses. Summary: It is necessary to precisely ascertain the risk of SARS-CoV-2 infection and the COVID-19 severity in IBD patients and to acknowledge the IBD management during the COVID-19 pandemic with clinically reliable information from COVID-19 cohorts and IBD experts' opinions. In this review, we highlight clinical questions regarding IBD management during the COVID-19 pandemic and make comments corresponding to each question based on recent publications. Key Messages: We propose that there is (1) no evidence that IBD itself increases the risk of SARS-CoV-2 infection, (2) to basically prioritize the control of karger@karger.com

www.karger.com/dig

Karger ${ }^{\prime}=$
(C) 2020 S. Karger AG, Base
Hiroshi Nakase

Department of Gastroenterology and Hepatology Sapporo Medical University School of Medicine S-1, W-16, Chuoku, Sapporo 060-8543 (Japan) hiro_nakase@sapmed.ac.jp 
disease activity of IBD, (3) no need for physicians to suddenly discontinue immunomodulatory or biologic therapy in patients with quiescent IBD, and (4) a need for careful observation of elderly ( $>60$ years old) and IBD patients receiving corticosteroid treatment during the COVID-19 pandemic.

(c) 2020 S. Karger AG, Basel

\section{Introduction}

Coronavirus infectious disease 2019 (COVID-19) is caused by severe acute respiratory syndrome coronavirus 2 (SARS-CoV-2) [1]. Detection of the virus was first reported in Wuhan, China, and has since spread worldwide [2]. Following the rapid spread of the virus across the globe, the World Health Organization (WHO) declared COVID-19 a pandemic on March 11, 2020 [3]. More than $10 \%$ of patients with COVID-19 need hospitalization with an increased need to have access to intensive care units and a mortality on the order of 3\% globally [3]. The number of confirmed cases of COVID-19 is still lower in Japan (15,663 cases as of May 10; https://coronavirus.jhu. edu/map.html); than in the USA and European countries, but the COVID-19 pandemic has impacted the healthcare system as well as the society in Japan. The Japanese government declared on April 16 a state of emergency all over Japan by reducing their mobility within prefectures and by avoiding across border crossing to slow the spread of COVID-19. Japanese hospitals have started to reduce elective activities of high-risk procedure such as endoscopy and surgery to mitigate viral spread, preserve personal protection equipment, and to be operationally ready for potential high numbers of COVID-19 admissions.

The number of patients with inflammatory bowel disease (IBD) is still increasing in Japan. Active IBD patients need systemic corticosteroids, thiopurine, and biologics for induction and maintenance of remission [4]. Data on COVID-19 in patients with immune-mediated inflammatory disease including IBD who have received anti-cytokine biologics, other immunomodulatory therapies, or both on a long-term basis are scarce. On the other hand, the SARS-CoV-2 infection is dynamic, and knowledge and evidence are growing rapidly. All gastroenterologists are facing clinical issues and dilemmas that they have never encountered before, and furthermore, the lack of robust evidence makes physicians' work even more challenging. In this regard, it is important to accumulate our knowledge on SARS-CoV-2 for the management of IBD patients. For IBD patients and for many physicians who are attending in the management of the disease in Japan, we have decided to collect and organize information regarding COVID-19 in the IBD field and to deliver it to the clinical field. Based on this idea, we constructed a team referred to as the "JAPAN IBD COVID-19 Taskforce" that started in April 2020. The main purposes of this taskforce are (a) to make proposals for IBD treatment in Japan based on transmission of literature information and (b) to summarize weekly summaries of data on the Surveillance Epidemiology of Coronavirus Under Research Exclusion (SECURE-IBD) database (https://covidibd. org/). In this article, we describe the clinical practice update incorporating the emerging understanding of COVID-19 and summarize available guidance for patients with IBD based on the most recent data accumulated until May 7, 2020.

\section{What Is the New Coronavirus Infection?}

The name of the new coronavirus is referred to as SARS-CoV-2, and when symptoms occur due to SARSCoV-2 infection, it is defined as the COVID-19. SARS$\mathrm{CoV}-2$ is an RNA virus, and its genomic sequence shares approximately $79 \%$ homology with the SARS virus (SARS-CoV) and 50\% homology with the MERS virus (MERS-CoV) [5]. SARS-CoV-2 is an enveloped, positively charged, single-stranded RNA virus belonging to the beta coronavirus genus. SARS-CoV-2 enters cells via the angiotensin-converting enzyme 2 (ACE2) expressed on the type II surfactant-secreting alveolar cells of the lung $[6,7]$, and it is highly homologous to SARS-CoV [8]. SARS-CoV-2 appears to be less fatal but more contagious than MERS-CoV and SARS-CoV [8]. Additionally, ACE2 is abundantly expressed in the glandular cells of gastric, duodenal, and rectal epithelia, supporting the entry of SARS-CoV-2 into host gastrointestinal (GI) cells [9]. The continuous positive detection of the viral RNA from feces thus suggests that the infectious virions are secreted from virus-infected GI cells [9] and implying possible fecaloral transmission of SARS-CoV-2 [10-12].

\section{GI Symptoms in COVID-19 and Their Relevance in the Management of IBD Patients}

Initial studies of COVID-19 reported that the proportion of patients presenting with GI symptoms was low $[13,14]$. After these reports, Tian et al. [15] reviewed the data of cases published as case reports or as retrospective 
clinical studies during a period from the end of December 2019 to the end of February 2020. According to this review, the frequency of GI symptoms varied depending on symptoms as follows: anorexia was the most frequent digestive system symptom in adults (39.9-50.2\%), diarrhea was the most common symptom both in adults and children (2-49.5\%), and vomiting was more common in children than in adults. Approximately 3.6-15.9\% of adult patients and $6.5-66.7 \%$ of children presented vomiting. Nausea was present in 1-29.4\%, GI bleeding was present in $4-13.7 \%$, and abdominal pain $(2.2-6.0 \%)$ was more frequent in severely ill patients than in nonseverely ill patients. Importantly, GI symptoms were more frequent in patients with severe COVID-19 than in those with nonsevere COVID-19. Most recently, Sultan et al. [16] reported that the overall prevalence of GI symptoms in COVID-19 was 7.7\% (95\% CI 7.4-8.6\%) for diarrhea, $7.8 \%$ (95\% CI 7.1-8.5\%) for nausea/vomiting, and 3.6\% (95\% CI $3.0-4.3 \%)$ for abdominal pain. Notably, the pooled prevalence of diarrhea is lower (4.0\%) in outpatients than in hospitalized patients. Based on these data, they suggested that GI symptoms are not as common in COVID-19 as previously estimated. Interestingly, in some cases, COVID-19 may present as isolated GI symptoms prior to the development of upper respiratory infection symptoms because diarrhea can precede other symptoms by a few days [16]. Taken together, physicians consider the possibility of COVID-19 when encountering patients who complain of new fever, cough, shortness of breath, or other upper respiratory infection symptoms after the onset of GI symptoms. Therefore, it seems to be difficult to differentiate diarrhea related to COVID-19 from a disease flare in IBD patients. Therefore, gastroenterologists should be aware of the following 2 possibilities during the COVID-19 pandemic [1]. The possibility of COVID-19 should be considered in IBD patients who develop unexpected GI symptoms such as diarrhea and vomiting even if they do not have fever or respiratory symptoms. If necessary, chest radiographs and chest computed tomography should be performed [2]. The risk of spreading SARS-CoV-2 should always be taken into consideration when performing lower GI endoscopy. Colonoscopies in patients with inactive IBD for the assessment of mucosal healing and surveillance of colorectal cancer should be avoided, although the risk of spreading SARS-CoV-2 by colonoscopy remains unclear. In symptomatic patients and for the diagnosis of treatment, physicians should carefully perform endoscopy with the protection against SARS-CoV-2, according to the guidelines [17].

\section{Risk of COVID-19 in IBD Patients}

In Japan, there have been an increasing number of patients with IBD treated with immunomodulators (IMs) or biologic agents. When the COVID-19 pandemic emerged, patients with IBD, especially those on systemic corticosteroids, thiopurines, and biologics, were considered to be moderate-to-high risk patients who are susceptible to COVID-19 and its complications. Therefore, both on-site physicians and IBD patients in Japan have concerns as to the justification of discontinuing their immunosuppressive therapies, including corticosteroids, IMs, and biologics during the COVID-19 outbreak as well as in other countries. Currently, there are no formal evidence-based recommendations from clinical societies or governments for IBD patients on immunosuppressive therapies during the COVID-19 pandemic. Additionally, there has been no evidence that IBD itself increases the risk of SARSCoV-2 infection. Moreover, the discontinuation of immunosuppressive treatments in IBD patients has not been recommended in guidance for patients proposed by the International Organization of IBD [18], Crohn's \& Colitis UK [19], or Crohn's \& Colitis Foundation [20] in their guidance for patients.

The SECURE-IBD (https://covidibd.org/) database provides international updates on COVID-19 in IBD patients including data from children and adults, and it is possible to know the number of IBD patients with COVID-19 according to the country, COVID-19 severity, age, and drug use status. According to the SECURE-IBD data [21], the mortality rate is high, especially in the elderly among the IBD patients who have developed COVID-19. COVID-19 is more severe in patients with ulcerative colitis (UC) than in those with Crohn's disease, although the reason for the different severity between the 2 diseases has not yet been clarified.

\section{Expert Proposals and Summaries for the Management of IBD Patients in Japan under the COVID-19 Pandemic Situation Based on the Transmission of Literature Information}

During the COVID-19 pandemic, we have come to consider several important questions regarding IBD management for on-site physicians and IBD patients. To answer these questions, Japan IBD COVID-19 Taskforce members collected up-to-date information regarding SARSCoV-2 and COVID-19 and summarized the available the information and guidance in a question-and-answer style: 
Q1. How is SARS-CoV-2 infection different from COVID-19?

A1. Generally, SARS-CoV-2 infection means an asymptomatic state, and COVID-19 means that symptoms due to SARS-CoV-2 infection have appeared.

Q2. Are patients with IBD at an increased risk of infection of SARS-CoV-2 infection and the development of COVID-19?

A2. At present, there has been no report showing a higher risk of COVID-19 in IBD patients than the general population. However, based on the SECURE-IBD data, patients receiving steroids tend to require elevated rates of hospitalization, ICU management, and ventilator usage. Therefore, we recommend that the unnecessary long-term administration of steroids should be avoided $[18,21,22]$.

Q3. Is COVID-19 likely to become more severe in elderly IBD patients?

A3. Based on the SECURE-IBD data, elderly IBD patients with COVID-19 tend to have elevated rates of hospitalization, ICU management, ventilator use, and mortality. In this regard, it is considered that as in the general population, the severity of COVID-19 in IBD patients increases with the age (particularly in patients older than 60 years) $[21,23,24]$.

Q4. Should all IMs and biologics treatments be discontinued?

A4. In clinical practice, the most important point is that the general condition of IBD patients is stable. Therefore, there is no need for physicians to abruptly discontinue immunomodulatory or biologic therapy in patients with quiescent IBD. Additionally, it is important that physicians explain to their patients that treatment should not be interrupted based on their own judgment. Rubin et al. [25] advocated the treatment guidance for IBD patients with concomitant SARS-CoV-2 infection or COVID-19. However, there is no established evidence yet. Currently, when IBD patients are diagnosed with COVID-19 [1], discontinuation of thiopurines, methotrexate, and JAK inhibitor [2] and extension of the interval of administration for biological drugs (extend 14 days from the scheduled date) are proposed [25-27].

Q5. What are the indications and precautions to be taken when performing endoscopy in IBD patients under the COVID-19 pandemic situation?

IBD Management during the COVID-19

Pandemic
A5. (a) SARS-CoV-2 RNA was detected in the feces. (b) ACE2, which is a SARS-CoV-2 entry receptor, is expressed in intestinal epithelial cells, and there is a possibility that the virus replication occurs in the intestinal epithelial cells $[28,29]$. Based on these points, it would be considered that SARS-CoV-2 may cause fecal-oral transmission.

Currently, it is proposed that in cases of patients with quiescent IBD (no abdominal symptoms), regular endoscopy for surveillance purposes should be postponed in all IBD patients. However, in the following conditions, even during the COVID-19 pandemic, there are cases where IBD patients need endoscopy under careful consideration of risks and benefits [30]: (1) for the diagnosis of patients with suspected IBD complaining of moderate to severe symptoms, (2) IBD patients with moderate to severe relapse, and (3) IBD patients who need to significantly escalate treatment.

On the other hand, in COVID-19 patients, approximately $3 \%$ of adults and $10 \%$ of children complain of GI symptoms without respiratory symptoms [15]. If GI symptoms occur in an IBD patient who has neither fever nor respiratory symptoms, carefully follow up the symptoms and consider possible COVID-19 by checking a chest radiograph and computed tomography before performing the endoscopy. When performing the endoscopy, medical staff and doctors should fully prevent infection with personal protection equipment.

Q6. What is the risk of COVID-19 becoming more severe in pregnant IBD patients?

A6. There are no direct reports regarding the risk of COVID-19 severity in IBD patients during pregnancy. However, we must acknowledge introducing references from overseas that are clinically useful.

A case series of 7 pregnant women with COVID-19 in New York was reported as follows: (a) 5 out of 7 had symptoms such as cough and fever and 4 were hospitalized. (b) Two asymptomatic cases developed postpartum and required treatment in the ICU [31]. Data from 118 pregnant women living in Wuhan who met the clinical criteria for COVID-19 pneumonia in China (from December 8, 2019 to March 20, 2020) were reported as follows: (a) $0.24 \%$ of pregnant women were infected, 109 (92\%) had mild, and 9 (8\%) had severe. (b) By March 20, 2020,109 patients (92\%) were discharged from the hospital. (c) Pregnant women had an equivalent risk of severe COVID-19 compared to that of the general population. (d) Of the 9 cases that became severe, 6 occurred postpartum [32]. Although few reports have been published to

Digestion 2021;102:814-822

817 
date, the results of these observational studies suggest that COVID-19 may become severe postpartum. Therefore, similar to that in the general public, if IBD patients during pregnancy suffer from SARS-CoV-2 infection or COVID-19, it may be necessary to pay attention to the postnatal severity.

\section{The Relationship between IBD Treatment and the Risk of SARS-CoV-2 Infection and the Severity of COVID-19}

There is no evidence supporting increases in the risk of SARS-CoV-2 infection or the risk of COVID-19 severity in IBD patients. However, it is considered that the risk of SARS-CoV-2 infection and the severity of COVID-19 in IBD patients may be associated with the disease activity of the underlying disease. Therefore, it is important to continue the current treatment to maintain remission in IBD patients as much as possible. In addition, for active patients, it is necessary to select the treatment to achieve prompt remission depending on the individual patient's condition and in consideration of the efficacy and safety of medications (please refer to Q2 and Q4).

Q7. Is 5-ASA administration related to COVID-19 risk and severity?

A7. To date, there have been no reports showing that 5-ASA increases the risk of bacterial or viral infection [33]. The use of 5-ASA will not increase the risk of SARSCoV-2 infection or the severity of COVID-19. Therefore, it is not necessary for IBD patients to discontinue 5-ASA use even if they are in close contact with COVID-19 patients or develop COVID-19 [34, 35].

Q8. Is the risk of the severity of COVID-19 increased in IBD patients receiving systemic steroids?

A8. In expectation of the anti-inflammatory effect, steroid administration was tried for SARS, MERS, and ARDS associated with influenza virus infection. However, the therapeutic effect of steroids remains unclear, and conversely, it was reported that the adverse events such as the need for ventilator management and the mortality increased in patients treated with steroids [36-38]. Additionally, there is a delay in the elimination of viral RNA from respiratory secretions in patients treated with steroids [39].

The use of more than $20 \mathrm{mg}$ of prednisolone was associated with an increased risk of respiratory infection in
IBD patients, as well as hospitalization and mortality [ 40 42]. However, there are no definitive reports of whether steroid administration affects the clinical outcome of COVID-19. Based on the current SECURE-IBD data, there is a trend that a higher proportion of IBD patients receiving steroid treatment resulted in ICU care, mechanical ventilation, or death than those receiving other medications [21]. However, the SECURE-IBD data are from a retrospective and observational study, and they do not directly indicate that the steroid use contributes to severe COVID-19. Therefore, a careful interpretation of these data is required.

During the COVID-19 pandemic, we propose the following:

1. Avoid simple use of systemic prednisolone and consider other alternative treatments as induction therapy.

2. However, if physicians choose systemic administration, a sufficient dose should be administered depending on the patients' disease activity.

3. For patients who are using systemic steroids, assess the therapeutic effect as soon as possible and try to taper the dose of systemic steroids rapidly.

Q9. Does the use of budesonide reduce the risk of severe of COVID-19 in comparison with that for systemic steroids?

A9. Budesonide is a steroid that acts locally in the intestine and has little systemic effect. Theoretically, the risk of infection is considered to be low during its administration [43, 44]. In reference to a review in Europe and the USA, if COVID-19 symptoms appear in IBD patients during steroid treatment or if the patients contact an infected person, physicians should consider a (a) prompt reduction in steroids and (b) switch treatment from steroids to budesonide if patients require the continuation of steroids [35]. However, the SECURE-IBD data show that even IBD patients treated with budesonide tend to have a more severe course of COVID-19 cases [21].

Therefore, during the COVID-19 pandemic, we propose the following:

1. Similar to systemic steroids, prolonged use of budesonide should be avoided. A reduction in dose should be promptly considered after patients achieve remission during induction therapy.

2. It remains unclear whether the risk of severe COVID-19 is reduced by the switch from systemic steroids to budesonide. 
Q10. Do IBD patients who take IMs have an increased risk of severe COVID-19?

A10. Thiopurines such as azathioprine and 6-mercaptopurine weaken the human immune response to viruses and have been suggested to be associated with an increased risk of viral infection after the administration [45]. On the other hand, there is little evidence of an increased risk of respiratory infections $[45,46]$. For example, MERS-CoV requires a protease during maturation, while in vitro studies have shown that mercaptopurine has an inhibitory effect on that protease [35]. However, no animal experiments have been conducted to verify its antiviral effect. Methotrexate, which is an IM, is also associated with an increased risk of infection [47]. However, it has been reported that methotrexate use does not increase the risk of infection in patients with inflammatory diseases [47]. Taken together, there is no clear evidence for an increased risk of respiratory tract infections with the use of thiopurines or methotrexate. Therefore, patients with clinically quiescent IBD do not need to discontinue IMs. Again, it is important to instruct patients to not readily discontinue IMs. Based on the SECUREIBD data, it is possible that COVID-19 is severe in IBD patients receiving IM treatment, following steroids and budesonide [21]. Although the patient backgrounds registered in the SECURE-IBD are obscure, it is necessary to pay attention to the white blood cell count (in particular, the decrease in lymphocytes) in patients receiving thiopurines.

During the COVID-19 pandemic, we proposed the following:

1. If IBD patients receiving IMs make contact with a COVID-19 patient, consider discontinuing administration of IMs for 2 weeks.

2. If IBD patients receiving IMs are diagnosed with SARS-CoV-2 infection/COVID-19, consider the discontinuation of IMs until the PCR results become negative for the virus.

Q11. Do IBD patients receiving biologics have an increased risk of severe COVID-19?

Anti-Tnfa Antibody

Patients treated with anti-TNFa antibody have an increased risk of respiratory infections and pneumonia, including tuberculosis, and the combined use with IMs further increases the risk [48-50]. On the other hand, anti-TNFa antibodies are used for the management of patients with severe sepsis, and it has been reported that administration of anti-TNFa antibodies before the onset of shock reduces subsequent mortality [51]. The blood cytokine pattern of COVID-19 is similar to that of secondary hemophagocytic lymphohistiocytosis, which is a hyperinflammatory syndrome, and it has also been reported that TNFa levels are elevated in the blood and local organs of COVID-19 patients [52, 53]. Therefore, clinical trials regarding the efficacy of anti-TNF antibodies to suppress excessive inflammation in COVID-19 patients have been proposed [53]. The SECURE-IBD data demonstrate that the total number of patients receiving anti-TNFa antibodies is the highest among the registered IBD patients with COVID-19, but the severity of COVID-19 in patients receiving antiTNF $\alpha$ antibodies is low [21]. When anti-TNFa antibodies are used in combination with IMs, COVID-19 severity is slightly increased. Thus, IBD patients with COVID-19during anti-TNF therapyalone may not deteriorate compared with those treated with other drugs. However, it should be noted that there are insufficient data to make any conclusions on the outcome of COVID-19. From the viewpoint reducing the risk of severe COVID-19, it may be possible to consider discontinuation of IMs in patients with quiescent IBD receiving a combination of anti-TNFa antibodies and IMs, especially those (a) whose endoscopic remission has already been achieved or (b) whose age is over $>60$ years.

Biologics Other Than anti-TNFa Antibodies

Based on the results of long-term administration studies, there is no report that anti-IL12/23p40 antibodies and vedolizumab increase the risk of serious infections, including respiratory infection [54-60]. Therefore, it is not necessary to consider discontinuing these drugs in IBD patients with clinical remission. The most recent SECURE-IBD data show a slightly increased hospitalization rate for patients receiving vedolizumab [21], but it is necessary to carefully consider the forthcoming data.

During the COVID-19 pandemic, we propose the following:

1. If patients receiving biologic therapy are in close contact with patients with COVID-19, consider postponing the biologics for 2 weeks from the scheduled date.

2. If patients receiving biologic therapy are diagnosed with SARS-CoV-2 infection/COVID-19, suspend the biologics until the PCR results become negative for the virus.

However, it should be noted that administration of anti-TNFa antibodies does not contribute to an increased risk of severe COVID-19.

Q12. Do IBD patients receiving JAK inhibitors have an increased risk of severe COVID-19? 
A12. The JAK inhibitor currently used in the IBD field is tofacitinib (TOF). TOF administration increases the risk of herpes zoster infection in patients with UC [61]. In particular, the risk is increased when $10 \mathrm{mg}$ of TOF is administered twice per day, in elderly individuals and in combination with steroids $[62,63]$. The SECURE-IBD data show that severe COVID-19 is frequent in IBD patients receiving JAK inhibitors, but it is important to carefully follow up and monitor patients treated with TOF because the number of cases registered in SECURE-IBD is small [21]. From the viewpoint of suppression of cytokine storms associated with COVID-19, it has been proposed that the continuation of JAK inhibitors, as well as anti-TNFa antibodies, may be acceptable [52]. We herein introduce the most recently reported case report [64]. A 33 -year-old female patient with UC who was refractory to 2 anti-TNFa antibodies and vedolizumab suffered from COVID-19 during treatment with $10 \mathrm{mg}$ of TOF twice per day. However, the authors continued the TOF at the same dose after she developed COVID-19. Her respiratory symptoms improved at 5 days after the onset of COVID-19 and her UC remained stable. The authors described that "starting a JAK inhibitor in the presence of SARS-CoV-2 infection or COVID-19 is not recommended, but it may be possible to consider continuing the treatment if COVID-19 develops during JAK inhibitor administration." This case indicates the significance of suppressing cytokine storms in patients with COVID-19. However, there are still few such cases, and we should be careful in continuing JAK inhibitor administration in IBD patients with COVID-19.

During the COVID-19 pandemic, we propose the following:

- The lowest dose to maintain remission should be used. If an IBD patient receiving $10 \mathrm{mg}$ of TOF twice daily is in a stable condition, consider reducing the dose to $5 \mathrm{mg}$ of TOF twice daily.

- If an IBD patient receiving TOF is in close contact with a COVID-19 patient, consider temporarily withholding TOF for 2 weeks.

- If an IBD patient receiving TOF is diagnosed with SARS-CoV-2 infection/COVID-19, suspend TOF until the virus become negative by PCR.

\section{Conclusion}

The COVID-19 pandemic represents the most challenging issue in the biologic era of IBD. IBD patients, medical staff, and on-site physicians worldwide have struggled tremendously in these difficult times. To overcome this pandemic situation and protect the quality of life of our patients, we must put all our efforts toward the best management of IBD based on clinical experience in the real world. Finally, we would like to emphasize that "there is no rain that does not stop" and hope that the critical pandemic calms soon.

\section{Conflict of Interest Statement}

Dr. Hiroshi Nakase (H.N.) reports receiving personsal fees from Abbvie Inc., Kissei Pharmaceutical Co., Ltd., Kyorin Pharmaceutical Co., Ltd., Mitsubishi Tanabe Pharma Corporation, Janssen Pharmaceutical K.K., Takeda Pharmaceutical Co., Ltd., Pfizer Lnc., Cell gene Corporation., EA Pharma Co., Ltd., Zeria Pharmaceutical Co., Ltd., Mochida Pharmaceutical Co., Ltd., Nippon Kayaku Co., Ltd., Daiichi Sankyo Co., Ltd., and JIMRO Co., Ltd., as well as grants for commissioned/joint research from the Hoya Group Pentax Medical, Boehringer Ingelheim GmbH, and Bristol Myers Squibb Co.

Dr. Takayuki Matsumoto (T.M.) reports receipt of personal fees from Abbvie Inc., Kissei Pharmaceutical Co., Ltd., Kyorin Pharmaceutical Co., Ltd., Janssen Pharmaceutical K.K, Takeda Pharmaceutical Co., Ltd., EA Pharma Co., Ltd., Mochida Pharmaceutical Co., Ltd., Nippon Kayaku Co., Ltd., Mitsubishi Tanabe Pharma Corporation, and JIMRO Co., Ltd.

Dr. Minoru Matsuura (M.M.) reports receipt of personal fees from AbbVie GK, Mitsubishi Tanabe Pharma Corporation, Nippon Kayaku Co., Ltd., Janssen Pharmaceutical K.K., Takeda Pharmaceutical Co. Ltd., Kyorin Pharmaceutical Co., Ltd., and Mochida Pharmaceutical Co., Ltd.

Dr. Hideki Iijima (H.I.) reports personal fees from AbbVie Inc, Janssen Pharmaceutical K.K., EA Pharma Co., Ltd., Eisai Pharmaceutical Co., Ltd., Mitsubishi Tanabe Pharma Co., Ltd., and Mochida Pharmaceutical Co. Ltd. and research grants from AbbVie Inc., Nippon Kayaku Co., Ltd, and Daiichi Sankyo Co., Ltd.

Dr. Katsuyoshi Matsuoka (K.M.) reports personal fees from AbbVie Inc, Kissei Pharmaceutical Co., Ltd., Mitsubishi Tanabe Pharma Co., Ltd., Janssen Pharmaceutical K.K., Takeda Pharmaceutical Co., Ltd., Pfizer Lnc., EA Pharma Co., Ltd., and Mochida Pharmaceutical Co., Ltd. and commercial research funding from Mitsubishi Tanabe Pharma Corporation, Mochida Pharmaceutical Co., Ltd., AbbVie GK, Nippon Kayaku Co., Ltd., and EA Pharma Co., Ltd.

Dr. Naoki Ohmiya (N.O.) reports receiving grants for commissioned/joint research from Nippon Kayaku Co., Ltd., EA Pharma Co., Ltd., and EA Pharma Co., Ltd.

Dr. Shunji Ishihara (S.I.) reports receipt of personal fees from Takeda Pharmaceutical Co. Ltd. and receiving grants for commissioned/joint research from Takeda Pharmaceutical Co. Ltd., Nippon Kayaku Co., Ltd., Astellas Pharma Inc., EA Pharma Co., Ltd., Zeria Pharmaceutical Co., Ltd., Daiichi Sankyo Co., Ltd., and JIMRO Co., Ltd.

Dr. Fumihito Hirai (F.H.) reports receipt of personal fees from AbbVie GK, EA Pharma Co., Ltd., Janssen Pharmaceutical K.K., Mochida Pharmaceutical Co., Ltd., Mitsubishi Tanabe Pharma Corporation, and Takeda Pharmaceutical Co. Ltd. 
Dr. Kouhei Wagatsuma (K.W.) and Yoshihiro Yokoyama (Y.Y.) report no receipt of personal fee from any pharmaceutical companies.

Dr. Tadakazu Hisamatsu (T.H.) reports receipt of personal fees from EA Pharma Co. Ltd., AbbVie GK, Celgene K.K., Janssen Pharmaceutical K.K., Pfizer Inc., Mitsubishi Tanabe Pharma Corporation, Kyorin Pharmaceutical Co. Ltd., JIMRO Co., Ltd., Mochida Pharmaceutical Co., Ltd., and Nichi-lko Pharmaceutical Co., Ltd. and commercial research funding from EA Pharma Co., Ltd., AbbVie GK, Daiichi-Sankyo Co., Ltd., Takeda Pharmaceutical Co. Ltd., Pfizer Inc., Mochida Pharmaceutical Co., Ltd., Nippon Kayaku Co., Ltd., Kyorin Pharmaceutical Co., Ltd., JIMRO Co., Ltd., Mochida Pharmaceutical Co., Ltd., and ZERIA Pharmaceutical Co., Ltd.

\section{Funding Sources}

This work was supported by Health and Labor Sciences Research Grants for research on intractable diseases from the Ministry of Health, Labor and Welfare of Japan (Investigation and Research for intractable Inflammatory Bowel Disease to H.N.) and Japan Society for the Promotion of Science (JSPS) Grants-in-Aid for Scientific Research (KAKENHI) Grant Number JP18H02799 (to H.N.).

\section{Author Contributions}

All authors contributed equally to the following: drafting of the manuscript and critical revision of the manuscript for important intellectual content.

\section{References}

1 Sungnak W, Huang N, Bécavin C, Berg M, Queen R, Litvinukova M, et al. SARS-CoV-2 entry factors are highly expressed in nasal epithelial cells together with innate immune genes. Nat Med. 2020;26(5):681-7.

2 Zhu N, Zhang D, Wang W, Li X, Yang B, Song $\mathrm{J}$, et al. A novel coronavirus from patients with pneumonia in China, 2019. N Engl J Med. 2020;382(8):727-33.

3 World Health Organization. Naming the coronavirus disease (COVID-19) and the virus that causes it. 2020.

4 Matsuoka K, Kobayashi T, Ueno F, Matsui T, Hirai F, Inoue N, et al. Evidence-based clinical practice guidelines for inflammatory bowel disease. J Gastroenterol. 2018;53(3):305-53.

5 Lu R, Zhao X, Li J, Niu P, Yang B, Wu H, et al. Genomic characterisation and epidemiology of 2019 novel coronavirus: implications for virus origins and receptor binding. Lancet. 2020;395(10224):565-74.

6 Wu F, Zhao S, Yu B, Chen YM, Wang W, Song ZG, et al. A new coronavirus associated with human respiratory disease in China. $\mathrm{Na}$ ture. 2020;579(7798):265-9.

7 Letko M, Marzi A, Munster V. Functional assessment of cell entry and receptor usage for SARS-CoV-2 and other lineage $B$ betacoronaviruses. Nat Microbiol. 2020;5(4):562-9.

8 Meo SA, Alhowikan AM, Al-Khlaiwi T, Meo IM, Halepoto DM, Iqbal M, et al. Novel coronavirus 2019-nCoV: prevalence, biological and clinical characteristics comparison with SARS-CoV and MERS-CoV. Eur Rev Med Pharmacol Sci. 2020;24(4):2012-9.

9 Xiao F, Tang M, Zheng X, Liu Y, Li X, Shan H. Evidence for gastrointestinal infection of SARS-CoV-2. Gastroenterology. 2020;158(6): 1831-3.e3.

10 Xu Y, Li X, Zhu B, Liang H, Fang C, Gong Y, et al. Characteristics of pediatric SARS-CoV-2 infection and potential evidence for persistent fecal viral shedding. Nat Med. 2020;26(4): 502-5.
11 Wang W, Xu Y, Gao R, Lu R, Han K, Wu G, et al. Detection of SARS-CoV-2 in different types of clinical specimens. JAMA. 2020;323: $1843-4$.

12 Yeo C, Kaushal S, Yeo D. Enteric involvement of coronaviruses: is faecal-oral transmission of SARS-CoV-2 possible? Lancet Gastroenterol Hepatol. 2020 Apr;5(4):335-7.

13 Chen N, Zhou M, Dong X, Qu J, Gong F, Han $\mathrm{Y}$, et al. Epidemiological and clinical characteristics of 99 cases of 2019 novel coronavirus pneumonia in Wuhan, China: a descriptive study. Lancet. 2020;395(10223):507-13.

14 Wang D, Hu B, Hu C, Zhu F, Liu X, Zhang J, et al. Clinical characteristics of 138 hospitalized patients with 2019 novel coronavirus-infected pneumonia in Wuhan, China. JAMA. 2020;323(11):1061-9.

15 Tian Y, Rong L, Nian W, He Y. Review article: gastrointestinal features in COVID-19 and the possibility of faecal transmission. Aliment Pharmacol Ther. 2020;51(9):843-51.

16 Sultan S, Lim JK, Atayar O, Davitkov P, Feuerstein JD, Siddique SM, et al. AGA institute rapid recommendations for gastrointestinal procedures during the COVID-19 pandemic. Gastroenterology. 2020 Apr 1.

17 Japan Gastroenterological Endoscopy Society. Recommendations for gastrointestinal endoscopy for new coronavirus infection (COVID-19) 4th revised edition.

18 IOIBD. IOIBD Update on COVID19 for Patients with Crohn's Disease and Ulcerative Colitis. IOIBD. 2020.

19 Available from: https://www.crohnsandcolitis.org.uk/news/coronavirus-covid-19-advice.

20 Available from: https://www.crohnscolitisfoundation.org/what-ibd-patients-shouldknow-about-2019-novel-coronavirus-covid-19.

21 Brenner EJ, Ungaro RC, Colombel JF, Kappelman MD. SECURE-IBD database public data update.
22 Norsa L, Indriolo A, Sansotta N, Cosimo P, Greco S, D'Antiga L. Uneventful course in IBD patients during SARS-CoV-2 outbreak in northern Italy gastroenterology. 2020 Apr 2.

23 3rd Interview COVID-19 ECCO Taskforce, published March 27, 2020

24 Mazza S, Sorce A, Peyvandi F, Vecchi M, Caprioli F. A fatal case of COVID-19 pneumonia occurring in a patient with severe acute ulcerative colitis. Gut. 2020;69(6):1148-9.

25 Rubin DT, Feuerstein JD, Wang AY, Cohen $\mathrm{RD}$. AGA clinical practice update on management of inflammatory bowel disease during the COVID-19 pandemic: expert commentary. Gastroenterology. 2020 Apr 10.

26 1st Interview COVID-19 ECCO Taskforce, published March 13, 2020.

27 5th Interview COVID-19 ECCO Taskforce, published April 14, 2020.

28 Wu Y, Guo C, Tang L, Hong Z, Zhou J, Dong $\mathrm{X}$, et al. Prolonged presence of SARS-CoV-2 viral RNA in faecal samples. Lancet Gastroenterol Hepatol. 2020;5(5):434-5.

29 Neurath MF. Covid-19 and immunomodulation in IBD. Gut. 2020 Jul;69(7):1335-42.

30 Iacucci M, Cannatelli R, Labarile N, Mao R, Panaccione R, Danese S, et al. Endoscopy in inflammatory bowel diseases during the COVID-19 pandemic and post-pandemic period. Lancet Gastroenterol Hepatol. 2020;5(6): 598-606.

31 Breslin N, Baptiste C, Miller R, Fuchs K, Goffman D, Gyamfi-Bannerman C, et al. COVID-19 in pregnancy: early lessons. Am J Obstet Gynecol MFM. 2020 May;2(2):100111.

32 Chen L, Li Q, Zheng D, Jiang H, Wei Y, Zou $\mathrm{L}$, et al. Clinical characteristics of pregnant women with covid-19 in wuhan, China. N Engl J Med. 2020 Apr 17.

33 Ransford RA, Langman MJ. Sulphasalazine and mesalazine: serious adverse reactions reevaluated on the basis of suspected adverse reaction reports to the committee on safety of medicines. Gut. 2002;51(4):536-9. 
34 Danese S, Cecconi M, Spinelli A. Management of IBD during the COVID-19 outbreak: resetting clinical priorities. Nat Rev Gastroenterol Hepatol. 2020;17(5):253-5.

35 Al-Ani A, Prentice R, Rentsch C, Johnson D, Ardalan Z, Heerasing N, et al. Prevention, diagnosis and management of COVID-19 in the inflammatory bowel disease patient. Aliment Pharmacol Ther. 2020 Apr 29.

36 Stockman LJ, Bellamy R, Garner P. SARS: systematic review of treatment effects. PLoS Med. 2006;3(9):e343.

37 Arabi YM, Mandourah Y, Al-Hameed F, Sindi AA, Al Mekhlafi GA, Hussein MA, et al. Corticosteroid therapy for critically ill patients with the middle east respiratory syndrome. Am J Respir Crit Care Med. 2018; 197(6):757-67.

38 Ni YN, Chen G, Sun J, Liang BM, Liang ZA. The effect of corticosteroids on mortality of patients with influenza pneumonia: a systematic review and meta-analysis. Crit Care. 2019;23(1):99.

39 Tinsley A, Navabi S, Williams ED, Liu G Kong L, Coates MD, et al. Increased risk of influenza and influenza-related complications among 140,480 patients with inflammatory bowel disease. Inflamm Bowel Dis. 2019; 25(2):369-76.

40 Long MD, Farraye FA, Okafor PN, Martin C, Sandler RS, Kappelman MD. Increased risk of pneumocystis jiroveci pneumonia among patients with inflammatory bowel disease. Inflamm Bowel Dis. 2013;19(5):1018-24.

41 Orlicka K, Barnes E, Culver EL. Prevention of infection caused by immunosuppressive drugs in gastroenterology. Ther Adv Chronic Dis. 2013;4(4):167-85.

42 Dorrington AM, Selinger CP, Parkes GC, Smith M, Pollok RC, Raine T. The historical role and contemporary use of corticosteroids in inflammatory bowel disease. J Crohns Colitis. 2020 Mar 14.

43 Sherlock ME, Seow $\mathrm{CH}$, Steinhart AH, Griffiths AM. Oral budesonide for induction of remission in ulcerative colitis. Cochrane Database Syst Rev. 2010;10(10):CD007698.

44 Benchimol EI, Seow CH, Otley AR, Steinhart $\mathrm{AH}$. Budesonide for maintenance of remission in Crohn's disease. Cochrane Database Syst Rev. 2009;1(1):CD002913.
45 Kirchgesner J, Lemaitre M, Carrat F, Zureik M, Carbonnel F, Dray-Spira R. Risk of serious and opportunistic infections associated with treatment of inflammatory bowel diseases. Gastroenterology. 2018;155(2):337-46.e10.

46 Seksik P, Cosnes J, Sokol H, Nion-Larmurier I, Gendre JP, Beaugerie L. Incidence of benign upper respiratory tract infections, HSV and HPV cutaneous infections in inflammatory bowel disease patients treated with azathioprine. Aliment Pharmacol Ther. 2009;29(10): 1106-13.

47 Conway R, Low C, Coughlan RJ, O'Donnell MJ, Carey JJ. Methotrexate use and risk of lung disease in psoriasis, psoriatic arthritis, and inflammatory bowel disease: systematic literature review and meta-analysis of randomised controlled trials. BMJ. 2015;350: h1269.

48 Shah ED, Farida JP, Siegel CA, Chong K, Melmed GY. Risk for overall infection with anti-TNF and anti-integrin agents used in IBD: a systematic review and meta-analysis. Inflamm Bowel Dis. 2017;23(4):570-7.

49 Singh S, Facciorusso A, Dulai PS, Jairath V, Sandborn WJ. Comparative risk of serious infections with biologic and/or immunosuppressive therapy in patients with inflammatory bowel diseases: a systematic review and meta-analysis. Clin Gastroenterol Hepatol. 2020;18(1):69-81.

50 Long MD, Farraye FA, Okafor PN, Martin C Sandler RS, Kappelman MD. Increased risk of pneumocystis jiroveci pneumonia among patients with inflammatory bowel disease. Inflamm Bowel Dis. 2013;19(5):1018-24.

51 Lv S, Han M, Yi R, Kwon S, Dai C, Wang R. Anti-TNF- $\alpha$ therapy for patients with sepsis: a systematic meta-analysis. Int J Clin Pract. 2014;68(4):520-8.

52 Mehta P, McAuley DF, Brown M, Sanchez E, Tattersall RS, Manson JJ. COVID-19: consider cytokine storm syndromes and immunosuppression. Lancet. 2020 Mar 28;395(10229): 1033-4.

53 Feldmann M, Maini RN, Woody JN, Holgate ST, Winter G, Rowland M, et al. Trials of antitumour necrosis factor therapy for COVID-19 are urgently needed. Lancet. 2020; 395(10234):1407-9.
54 Feagan BG, Sandborn WJ, Gasink C, Jacobstein D, Lang Y, Friedman JR, et al. Ustekinumab as induction and maintenance therapy for Crohn's disease. N Engl J Med. 2016;375(20):1946-60

55 Kalb RE, Fiorentino DF, Lebwohl MG, Toole J, Poulin Y, Cohen AD, et al. Risk of serious infection with biologic and systemic treatment of Psoriasis: results from the Psoriasis longitudinal assessment and registry (PSOLAR). JAMA Dermatol. 2015;151(9):961-9.

56 Hanauer SB, Sandborn WJ, Feagan BG, Gasink C, Jacobstein D, Zou B, et al. IM-UNITI: three-year efficacy, safety, and immunogenicity of ustekinumab treatment of Crohn's disease. J Crohns Colitis. 2020;14(1):23-32.

57 Bye WA, Jairath V, Travis SPL. Systematic review: the safety of vedolizumab for the treatment of inflammatory bowel disease. Aliment Pharmacol Ther. 2017;46(1):3-15.

58 Mocko P, Kawalec P, Pilc A. Safety profile of biologic drugs in the therapy of Crohn disease: a systematic review and network meta-analysis. Pharmacol Rep. 2016;68(6):1237-43.

59 Mocko P, Kawalec P, Pilc A. Safety profile of biologic drugs in the therapy of ulcerative colitis: a systematic review and network metaanalysis. Pharmacotherapy. 2016;36(8):870-

60 Colombel JF, Sands BE, Rutgeerts P, Sandborn W, Danese S, D'Haens G, et al. The safety of vedolizumab for ulcerative colitis and Crohn's disease. Gut. 2017;66(5):839-51.

61 Winthrop KL, Melmed GY, Vermeire S, Long MD, Chan G, Pedersen RD, et al. Herpes zoster infection in patients with ulcerative colitis receiving tofacitinib. Inflamm Bowel Dis. 2018;24(10):2258-65.

62 Harigai M. Growing evidence of the safety of JAK inhibitors in patients with rheumatoid arthritis. Rheumatology. 2019;58(Suppl 1): i34-42.

63 Weisshof R, Aharoni Golan M, Sossenheimer $\mathrm{PH}$, El Jurdi K, Ollech JE, Pekow J, et al. Realworld experience with tofacitinib in IBD at a tertiary center. Dig Dis Sci. 2019;64(7):194551.

64 Jacobs J, Clark-Snustad K, Lee S. Case report of a SARS-CoV-2 infection in a patient with ulcerative colitis on tofacitinib. Inflamm Bowel Dis. 2020 Jun 18;26(7):e64. 